\title{
Genetic Drift
}

National Human Genome Research Institute (NHGRI)

\section{Source}

National Human Genome Research Institute (NHGRI). Genetic Drift.

Genetic drift is a mechanism of evolution. It refers to random fluctuations in the frequencies of alleles from generation to generation due to chance events. Genetic drift can cause traits to be dominant or disappear from a population. The effects of genetic drift are most pronounced in small populations. 